\title{
Research Article \\ Some Properties of Orthogonal Polynomials for Laguerre-Type Weights
}

\author{
HeeSun Jung ${ }^{1}$ and Ryozi Sakai ${ }^{2}$ \\ ${ }^{1}$ Department of Mathematics Education, Sungkyunkwan University, Seoul 110-745, Republic of Korea \\ ${ }^{2}$ Department of Mathematics, Meijo University, Nagoya 468-8502, Japan
}

Correspondence should be addressed to HeeSun Jung, hsun90@skku.edu

Received 3 November 2010; Accepted 21 February 2011

Academic Editor: P. Y. H. Pang

Copyright (C) 2011 H. Jung and R. Sakai. This is an open access article distributed under the Creative Commons Attribution License, which permits unrestricted use, distribution, and reproduction in any medium, provided the original work is properly cited.

Let $\mathbb{R}^{+}=[0, \infty)$, let $R: \mathbb{R}^{+} \rightarrow \mathbb{R}^{+}$be a continuous, nonnegative, and increasing function, and let $p_{n, \rho}(x)$ be the orthonormal polynomials with the weight $w_{\rho}(x)=x^{\rho} e^{-R(x)}, \rho>-1 / 2$. For the zeros $\left\{x_{k, n, \rho}\right\}_{k=1}^{n}$ of $p_{n, \rho}(x)=p_{n}\left(w_{\rho}^{2} ; x\right)$, we estimate $p_{n, \rho}^{(j)}\left(x_{k, n, \rho}\right)$, where $j$ is a positive integer. Moreover, we investigate the various weighted $L_{p}$-norms $(0<p \leqslant \infty)$ of $p_{n, \rho}(x)$.

\section{Introduction and Main Results}

Let $\mathbb{R}=(-\infty, \infty)$ and $\mathbb{R}^{+}=[0, \infty)$. Let $R: \mathbb{R}^{+} \rightarrow \mathbb{R}^{+}$be a continuous, nonnegative, and increasing function. Consider the exponential weights $w_{\rho}(x)=x^{\rho} \exp (-R(x)), \rho>-1 / 2$, and then we construct the orthonormal polynomials $\left\{p_{n, \rho}(x)\right\}_{n=0}^{\infty}$ with the weight $w_{\rho}(x)$. In this paper, for the zeros $\left\{x_{k n}\right\}_{k=1}^{n}$ of $p_{n, \rho}(x)=p_{n}\left(w_{\rho}^{2} ; x\right)$ we estimate $p_{n, \rho}^{(j)}\left(x_{k n}\right)$, where $j$ is a positive integer. Moreover, we investigate the various weighted $L_{p}$-norms $(0<p \leqslant \infty)$ of $p_{n, \rho}(x)$.

We say that $f: \mathbb{R} \rightarrow \mathbb{R}^{+}$is quasi-increasing if there exists $C>0$ such that $f(x) \leqslant C f(y)$ for $0<x<y$. The notation $f(x) \sim g(x)$ means that there are positive constants $C_{1}, C_{2}$ such that for the relevant range of $x, C_{1} \leqslant f(x) / g(x) \leqslant C_{2}$. The similar notation is used for sequences and sequences of functions.

Throughout, $C, C_{1}, C_{2}, \ldots$ denote positive constants independent of $n, x, t$. The same symbol does not necessarily denote the same constant in different occurrences. We denote the class of polynomials with degree $n$ by $p_{n}$.

First, we introduce some classes of weights.

Levin and Lubinsky $[1,2]$ introduced the class of weights on $\mathbb{R}^{+}$as follows. Let $I=$ $[0, d)$, where $0<d \leqslant \infty$. 
Definition 1.1 (see $[1,2]$ ). We assume that $R: I \rightarrow[0, \infty)$ has the following properties. Let $Q(t)=R\left(t^{2}\right)$,

(a) $\sqrt{x} R(x)$ is continuous in $I$, with limit 0 at 0 and $R(0)=0$,

(b) $R^{\prime \prime}(x)$ exists in $(0, d)$, while $Q^{\prime \prime}(t)$ is positive in $(0, \sqrt{d})$,

(c)

$$
\lim _{x \rightarrow d_{-}} R(x)=\infty
$$

(d) the function

$$
T(x):=\frac{x R^{\prime}(x)}{R(x)}
$$

is quasi-increasing in $(0, d)$, with

$$
T(x) \geqslant \Lambda>\frac{1}{2}, \quad x \in(0, d)
$$

(e) there exists $C_{1}>0$ such that

$$
\frac{\left|R^{\prime \prime}(x)\right|}{R^{\prime}(x)} \leqslant C_{1} \frac{R^{\prime}(x)}{R(x)}, \quad \text { a.e. } x \in(0, d)
$$

Then, we write $w \in \mathcal{L}\left(C^{2}\right)$. If there also exists a compact subinterval $J^{*} \ni 0$ of $I^{*}=(-\sqrt{d}, \sqrt{d})$, and $C_{2}>0$ such that

$$
\frac{Q^{\prime \prime}(t)}{\left|Q^{\prime}(t)\right|} \geqslant C_{2} \frac{\left|Q^{\prime}(t)\right|}{Q(t)}, \quad \text { a.e. } t \in I^{*} \backslash J^{*},
$$

then we write $w \in \mathcal{L}\left(C^{2}+\right)$.

We consider the case $d=\infty$, that is, the space $\mathbb{R}^{+}=[0, \infty)$, and we strengthen Definition 1.1 slightly.

Definition 1.2. We assume that $R: \mathbb{R}^{+} \rightarrow \mathbb{R}^{+}$has the following properties:

(a) $R(x), R^{\prime}(x)$ are continuous, positive in $\mathbb{R}^{+}$, with $R(0)=0, R^{\prime}(0)=0$,

(b) $R^{\prime \prime}(x)>0$ exists in $\mathbb{R}^{+} \backslash\{0\}$,

(c)

$$
\lim _{x \rightarrow \infty} R(x)=\infty,
$$

(d) the function 
Journal of Inequalities and Applications

$$
T(x):=\frac{x R^{\prime}(x)}{R(x)}
$$

is quasi-increasing in $\mathbb{R}^{+} \backslash\{0\}$, with

$$
T(x) \geqslant \Lambda>\frac{1}{2}, \quad x \in \mathbb{R}^{+} \backslash\{0\},
$$

(e) there exists $C_{1}>0$ such that

$$
\frac{R^{\prime \prime}(x)}{R^{\prime}(x)} \leqslant C_{1} \frac{R^{\prime}(x)}{R(x)}, \quad \text { a.e. } x \in \mathbb{R}^{+} \backslash\{0\}
$$

There exists a compact subinterval $J \ni 0$ of $\mathbb{R}^{+}$, and $C_{2}>0$ such that

$$
\frac{R^{\prime \prime}(x)}{R^{\prime}(x)} \geqslant C_{2} \frac{R^{\prime}(x)}{R(x)}, \quad \text { a.e. } x \in \mathbb{R}^{+} \backslash J
$$

then we write $w \in \mathcal{L}_{2}$.

Let us consider the weight $w \in \mathcal{L}\left(C^{2}+\right)$ in Definition 1.2. Levin and Lubinsky [2, Theorem 1.3] have given the following theorem.

Theorem A (see [3, Theorem 1.3]). Let $w \in \mathcal{L}\left(C^{2}+\right)$ and $\rho>-1 / 2$. There exists $n_{0}$ such that uniformly for $n \geqslant n_{0}, 1 \leqslant j \leqslant n$,

$$
\left|p_{n, \rho}^{\prime} w_{\rho}\right|\left(x_{j n}\right) \sim \varphi_{n}\left(x_{j n}\right)^{-1}\left[x_{j n}\left(a_{n}-x_{j n}\right)\right]^{-1 / 4}
$$

Now, we will estimate the higher-order derivatives of the orthonormal polynomials $p_{n}\left(w_{\rho}^{2} ; x\right)$. However, we need to focus on a smaller class of weights.

Definition 1.3. Let $w=\exp (-R) \in \mathcal{L}_{2}$ and $v \geqslant 2$ be an integer. For the exponent $R$, we assume the following:

(a) $R^{(j)}(x)>0$, for $0 \leqslant j \leqslant v$ and $x>0$, and $R^{(j)}(0)=0,0 \leqslant j \leqslant v-1$.

(b) there exist positive constants $C_{i}>0$ such that for $i=1,2, \ldots, v-1$

$$
R^{(i+1)}(x) \leqslant C_{i} R^{(i)}(x) \frac{R^{\prime}(x)}{R(x)}, \quad \text { a.e. } x \in \mathbb{R}^{+} \backslash\{0\},
$$

(c) there exist positive constants $C, 0<c_{1} \leqslant 1$ and $0 \leqslant \delta<1$ such that for $x \in\left(0, c_{1}\right)$

$$
R^{(v)}(x) \leqslant C\left(\frac{1}{x}\right)^{\delta}
$$


(d) there exists $c_{2}>0$ such that we have one among the following:

(d1) $T(x) / \sqrt{x}$ is quasi-increasing on $\left(c_{2}, \infty\right)$,

(d2) $R^{(v)}(x)$ is nondecreasing on $\left(c_{2}, \infty\right)$.

Then, we write $w(x)=e^{-R(x)} \in \tilde{\mathcal{L}}_{v}$.

Example 1.4 (see $[1,4]$ ). Let $v \geqslant 2$ be a fixed integer. There are some typical examples satisfying all conditions of Definition 1.3 constructed as follows: let $\alpha>1, l \geqslant 1$, where $l$ is an integer. Then, we define

$$
R_{l, \alpha}(x)=\exp _{l}\left(x^{\alpha}\right)-\exp _{l}(0)
$$

where $\exp _{l}(x)=\exp (\exp (\exp \cdots \exp (x)) \cdots)$ is the $l$ th iterated exponential.

(1) When $\alpha>v$, we consider $w(x)=e^{-R_{l, \alpha}(x)}$, then $w \in \tilde{\mathcal{L}}_{v}$.

(2) When $\alpha \leqslant v, \alpha$ is an integer, we define

$$
R_{l, \alpha}(x)=\exp _{l}\left(|x|^{\alpha}\right)-\exp _{l}(0)-\sum_{j=1}^{r} \frac{R_{l, \alpha}^{(j)}(0)}{j !} x^{j} .
$$

Then, $w(x)=e^{-R_{l, \alpha}(x)} \in \widetilde{\mathcal{L}}_{\nu}$.

In the rest of this paper, we consider the classes $\mathcal{L}_{2}$ and $\tilde{\mathcal{L}}_{v}$; let $w \in \mathcal{L}_{2}$ or $w \in \tilde{\mathcal{L}}_{v}$ $(v \geqslant 2)$. For $\rho>-1 / 2$, we set $w_{\rho}(x):=x^{\rho} w(x)$. Then, we can construct the orthonormal polynomials $p_{n, \rho}(x)=p_{n}\left(w_{\rho}^{2} ; x\right)$ of degree $n$ with respect to $w_{\rho}^{2}(x)$. That is,

$$
\int_{0}^{\infty} p_{n, \rho}(u) p_{m, \rho}(u) w_{\rho}^{2}(u) d u=\delta_{n m} \quad(\text { Kronecker's delta }) n, m=0,1,2, \ldots
$$

Let us denote the zeros of $p_{n, \rho}(x)$ by

$$
0<x_{n, n, \rho}<\cdots<x_{2, n, \rho}<x_{1, n, \rho}<\infty
$$

The Mhaskar-Rahmanov-Saff numbers $a_{v}$ are defined as follows:

$$
v=\frac{1}{\pi} \int_{0}^{1} a_{v} t R^{\prime}\left(a_{v} t\right)\{t(1-t)\}^{-1 / 2} d t, \quad v>0
$$

In this paper, we will consider the orthonormal polynomials $p_{n, \rho}(x)$ with respect to the weight class $\widetilde{\mathcal{L}}_{v}$. Our main themes in this paper are to estimate the higher-order derivatives of $p_{n, \rho}(x)$ at the zeros of $p_{n, \rho}(x)$ and to investigate the various weighted $L_{p}$-norms $(0<p \leqslant$ $\infty)$ of $p_{n, \rho}(x)$. More precisely, we will estimate the higher-order derivatives of $p_{n, \rho}(x)$ at all zeros of $p_{n, \rho}(x)$ for two cases of an odd order and of an even order. In addition, we will give 
asymptotic relation of the odd order derivatives of $p_{n, \rho}(x)$ at the zeros of $p_{n, \rho}(x)$ in a certain finite interval. These estimations will play an important role in investigating convergence or divergence of higher-order Hermite-Fejér interpolation polynomials (see [3, 5-17]).

Then, our main purpose is to obtain estimations with respect to $p_{n, \rho}^{(j)}\left(x_{k, n, \rho}\right), k=$ $1,2, \ldots, n, j=1,2, \ldots, v$ as follows.

Theorem 1.5. Let $w(x)=\exp (-R(x)) \in \mathcal{L}\left(C^{2}+\right)$ and $\rho>-1 / 2$. For each $k=1,2, \ldots, n$ and $j=0,1, \ldots, v-1$ one has

$$
\left|p_{n, \rho}^{(j)}\left(x_{k, n, \rho}\right)\right| \leqslant C\left(\frac{n}{\sqrt{a_{2 n}-x_{k, n, \rho}}}\right)^{j-1} x_{k, n, \rho}^{-(j-1) / 2}\left|p_{n, \rho}^{\prime}\left(x_{k, n, \rho}\right)\right| .
$$

Theorem 1.6. Let $w(x)=\exp (-R(x)) \in \tilde{\mathcal{L}}_{v}$ and $\rho>-1 / 2$. Assume that $1+2 \rho-\delta / 2 \geqslant 0$ for $\rho<-1 / 4$, and if $T(x)$ is bounded, then assume that

$$
a_{n} \leqslant C n^{2 /(1+v-\delta)}
$$

where $0 \leqslant \delta<1$ is defined in (1.13). For each $k=1,2, \ldots, n$ and $j=0,1, \ldots, v-1$, one has

$$
\left|p_{n, \rho}^{(j)}\left(x_{k, n, \rho}\right)\right| \leqslant C\left(\frac{n}{\sqrt{a_{2 n}}-\sqrt{x_{k, n, \rho}}}+\frac{T\left(a_{n}\right)}{\sqrt{a_{n}}}\right)^{j-1} x_{k, n, \rho}^{-(j-1) / 2}\left|p_{n, \rho}^{\prime}\left(x_{k, n, \rho}\right)\right|,
$$

and in particular if $j$ is even, then

$$
\begin{aligned}
\left|p_{n, \rho}^{(j)}\left(x_{k, n, \rho}\right)\right| \leqslant & C\left(\frac{T\left(a_{n}\right)}{\sqrt{a_{n} x_{k, n, \rho}}}+R^{\prime}\left(x_{k, n, \rho}\right)+\frac{1}{x_{k, n, \rho}}\right) \\
& \times\left(\frac{n}{\sqrt{a_{2 n}}-\sqrt{x_{k, n, \rho}}}+\frac{T\left(a_{n}\right)}{\sqrt{a_{n}}}\right)^{j-2} x_{k, n, \rho}^{-(j-2) / 2}\left|p_{n, \rho}^{\prime}\left(x_{k, n, \rho}\right)\right| .
\end{aligned}
$$

Theorem 1.7. Let $w(x)=\exp (-R(x)) \in \tilde{\mathcal{L}}_{v}$ and $\rho>-1 / 2$. Let $(1 / \varepsilon)\left(a_{n} / n^{2}\right) \leqslant x_{k, n, \rho} \leqslant \varepsilon a_{n}$, $0<\varepsilon<1 / 4$, and $v=2,3, \ldots, s=0,1, \ldots,(v-1) / 2$. Then, under the same conditions as the assumptions of Theorem 1.6, there exist $\beta(n, k), 0<D_{1} \leqslant \beta(n, k) \leqslant D_{2}$ for absolute constants $D_{1}$, $D_{2}$ such that the following equality holds:

$$
p_{n, \rho}^{(2 s+1)}\left(x_{k, n, \rho}\right)=(-1)^{s} \beta_{2 n}^{s}(2 n, k)\left(\frac{n}{\sqrt{a_{n}}}\right)^{2 s}\left(1+\rho_{s}\left(\varepsilon, x_{k, n, \rho}, n\right)\right) p_{n}^{\prime}\left(x_{k, n, \rho}\right) x_{k, n, \rho}^{-s}
$$

and $\left|\rho_{s}\left(\varepsilon, x_{k, n, \rho}, n\right)\right| \rightarrow 0$ as $n \rightarrow \infty$ and $\varepsilon \rightarrow 0$. 
Define

$$
\begin{gathered}
\Phi_{n}(x)=\max \left\{\eta_{n}, 1-\left(\frac{x}{a_{n}}\right)^{1 / 2}\right\}, \quad \eta_{n}=\left\{n T\left(a_{n}\right)\right\}^{-2 / 3}, \\
z^{+}= \begin{cases}z, & z>0, \\
0, & z \leqslant 0 .\end{cases}
\end{gathered}
$$

Let us define

$$
\Theta_{n}(x)=\frac{x / a_{n}}{1+x / a_{n}}
$$

We consider the class of weights, $\mathcal{F}\left(C^{2}\right)$, which is defined in Definition 2.1 below. Levin and Lubinsky have obtained the following theorem.

Theorem B (see [18, Theorem 13.6]). Assume that $W \in \mathcal{F}\left(C^{2}\right)$. Let $0<p<\infty$. Then uniformly for $n \geqslant 1$,

$$
\left\|P_{n} W\right\|_{L_{p}(I)} \sim \begin{cases}a_{n}^{1 / p-1 / 2}, & p<4 \\ a_{n}^{-1 / 4}(\log (n+1))^{1 / 4}, & p=4 \\ a_{n}^{1 / p-1 / 2}\left(n T\left(a_{n}\right)\right)^{(2 / 3)(1 / 4-1 / p)}, & p>4\end{cases}
$$

We remark that Levin and Lubinsky have shown Theorem B for more wider class $\mathcal{F}(\operatorname{lip}(1 / 2)) \supseteq \mathcal{F}\left(C^{2}\right)$. In the following, we investigate the various weighted $L_{p}$-norms $(0<$ $p \leqslant \infty)$ of $p_{n, \rho}(x)$.

Theorem 1.8. Let $w \in \mathcal{L}_{2}$. Let $0<p<\infty$ and $\rho>-1 / 2$. Then one has for $n \geqslant 1$,

$$
\left\|\Theta_{n}^{1 / 4}(x) p_{n, \rho}(x) w(x)\left(x+\frac{a_{n}}{n^{2}}\right)^{\rho}\right\|_{L_{p}\left(\mathbb{R}^{+}\right)} \sim a_{n}^{1 / p-1 / 2} \begin{cases}1, & p<4 \\ \left(\log \left(1+n T\left(a_{n}\right)\right)\right)^{1 / 4}, & p=4 \\ \left(n T\left(a_{n}\right)\right)^{(2 / 3)(1 / 4-1 / p)}, & p>4 .\end{cases}
$$

Theorem 1.9. Let $w \in \mathcal{L}_{2}$. Let $0<p<\infty$ and $\rho>-1 / 2$. Then one has for $n \geqslant 1$,

$$
\left\|p_{n, \rho}(x) w(x)\left(x+\frac{a_{n}}{n^{2}}\right)^{\rho}\right\|_{L_{p}\left(\mathbb{R}^{+}\right)} \sim a_{n}^{1 / p-1 / 2} \begin{cases}1, & p<4 \\ (\log n)^{1 / 4}, & p=4 \\ n^{2(1 / 4-1 / p)}, & p>4 .\end{cases}
$$


Theorem 1.10. Let $w \in \mathcal{L}_{2}, 0 \leqslant s \leqslant r$, and $n \geqslant 1$. Suppose that $\rho>-1 / 2$. For $0 \leqslant s \leqslant r$ and $n \geqslant 1$, one has

$$
\left\|\Theta_{n}^{r / 4}(x) \Phi_{n}^{(r / 4-1 / p)^{+}}(x)\left|p_{n, \rho}(x) w(x)\left(x+\frac{a_{n}}{n}\right)^{\rho}\right|^{s}\right\|_{L_{p}\left(\mathbb{R}^{+}\right)} \sim \begin{cases}a_{n}^{1 / p-r / 2} \log n, & \text { if } s=r, p r \geqslant 4 \\ a_{n}^{1 / p-s / 2}, & \text { otherwise, }\end{cases}
$$

and for $p=\infty$

$$
\left\|\Theta_{n}^{r / 4}(x) \Phi_{n}^{(r / 4-1 / p)^{+}}(x)\left|p_{n, \rho}(x) w(x)\left(x+\frac{a_{n}}{n}\right)^{\rho}\right|^{s}\right\|_{L_{p}\left(\mathbb{R}^{+}\right)} \sim a_{n}^{-s / 4}
$$

This paper is organized as follows. In Section 2, we will introduce the weight class $\tilde{F}_{v}$ as an analogy of the class $\tilde{\mathcal{L}}_{v}$ and the known results of orthonormal polynomials with respect to $\tilde{\mp}_{v}$ in order to prove the main results. In Section 3, we will prove Theorems 1.5, 1.6, and 1.7. Finally, we will prove the results for the various weighted $L_{p}$-norms $(0<p \leqslant \infty)$ of $p_{n, p}(x)$, that is, Theorems 1.8, 1.9, and 1.10, in Section 4.

\section{Preliminaries}

Levin and Lubinsky introduced the classes $\mathcal{L}\left(C^{2}\right)$ and $\mathcal{L}\left(C^{2}+\right)$ as an analogy of the classes $\mathcal{F}\left(C^{2}\right)$ and $\mathcal{f}\left(C^{2}+\right)$ which they already defined on $I^{*}=(-\sqrt{d}, \sqrt{d})$. They defined the following.

Definition 2.1 (see [18]). We assume that $Q: I^{*} \rightarrow[0, \infty)$ has the following properties:

(a) $Q(t)$ is continuous in $I^{*}$, with $Q(0)=0$,

(b) $Q^{\prime \prime}(t)$ exists and is positive in $I^{*} \backslash\{0\}$,

(c)

$$
\lim _{t \rightarrow \sqrt{d}-} Q(t)=\infty
$$

(d) the function

$$
T^{*}(t):=\frac{t Q^{\prime}(t)}{Q(t)}
$$

is quasi-increasing in $(0, \sqrt{d})$, with

$$
T^{*}(t) \geqslant \Lambda^{*}>1, \quad t \in I^{*} \backslash\{0\},
$$


(e) there exists $C_{1}>0$ such that

$$
\frac{Q^{\prime \prime}(t)}{\left|Q^{\prime}(t)\right|} \leqslant C_{1} \frac{\left|Q^{\prime}(t)\right|}{Q(t)}, \quad \text { a.e. } t \in I^{*} \backslash\{0\}
$$

Then, we write $W \in \mathcal{F}\left(C^{2}\right)$. If there also exists a compact subinterval $J^{*} \ni 0$ of $I^{*}$, and $C_{2}>0$ such that

$$
\frac{Q^{\prime \prime}(t)}{\left|Q^{\prime}(t)\right|} \geqslant C_{2} \frac{\left|Q^{\prime}(t)\right|}{Q(t)}, \quad \text { a.e. } t \in I^{*} \backslash J^{*},
$$

then, we write $W \in \mathcal{F}\left(C^{2}+\right)$.

Then we see that $w \in \mathcal{L}\left(C^{2}\right) \Leftrightarrow \mathcal{F}\left(C^{2}\right)$ and $w \in \mathcal{L}\left(C^{2}+\right) \Leftrightarrow \mathcal{F}\left(C^{2}+\right)$ from $[1$, Lemma 2.2]. In addition, we easily have the following.

Lemma 2.2. Let $Q(t)=R\left(t^{2}\right), x=t^{2}$. Then one has

$$
w \in \mathcal{L}_{2} \Longrightarrow W \in \mathcal{F}\left(C^{2}+\right)
$$

where $W(t)=w(x), x=t^{2}$.

On $\mathbb{R}$, we can consider the corresponding class to $\tilde{\mathcal{L}}_{v}$ as follows.

Definition 2.3 (cf. [19]). Let $W=\exp (-Q) \in \mathcal{F}\left(C^{2}+\right)$ and $v \geqslant 2$ be an integer. Let $Q$ be a continuous and even function on $\mathbb{R}$. For the exponent $Q$, we assume the following:

(a) $Q^{(j)}(t)>0$, for $0 \leqslant j \leqslant v$ and $t \in \mathbb{R}^{+} \backslash\{0\}$,

(b) there exist positive constants $C_{i}>0$ such that for $i=1,2, \ldots, v-1$

$$
Q^{(i+1)}(t) \leqslant C_{i} Q^{(i)}(t) \frac{Q^{\prime}(t)}{Q(t)}, \quad \text { a.e. } x \in \mathbb{R}^{+} \backslash\{0\}
$$

(c) there exist positive constants $C, c_{1}>0$ and $0 \leqslant \delta^{*}<1$ such that for $t \in\left(0, c_{1}\right)$

$$
Q^{(v)}(t) \leqslant C\left(\frac{1}{t}\right)^{\delta^{*}}
$$

(d) there exists $c_{2}>0$ such that one has one among the following:

(d1) $T^{*}(t) / t$ is quasi-increasing on $\left(c_{2}, \infty\right)$,

$(\mathrm{d} 2) Q^{(v)}(t)$ is nondecreasing on $\left(c_{2}, \infty\right)$.

Then, we write $W(t)=e^{-Q(t)} \in \tilde{\mathscr{F}}_{\nu}$. 
Journal of Inequalities and Applications

Let $W \in \tilde{\Psi}_{v}$ and $v \geqslant 2$. For $\rho^{*}>-1 / 2$, we set

$$
W_{\rho^{*}}(t):=|t|^{\rho^{*}} W(t)
$$

Then, we can construct the orthonormal polynomials $P_{n, \rho^{*}}(t)=P_{n}\left(W_{\rho^{*}}^{2} ; t\right)$ of degree $n$ with respect to $W_{\rho^{*}}(t)$. That is,

$$
\int_{-\infty}^{\infty} P_{n, \rho^{*}}(v) P_{m, \rho^{*}}(v) W_{\rho^{*}}^{2}(v) d t=\delta_{n m}, \quad n, m=0,1,2, \ldots
$$

Let us denote the zeros of $P_{n, \rho^{*}}(t)$ by

$$
-\infty<t_{n n}<\cdots<t_{2 n}<t_{1 n}<\infty .
$$

Jung and Sakai $\left[5\right.$, Theorems 3.3 and 3.6] estimate $P_{n, \rho^{*}}^{(j)}\left(t_{k, n}\right), k=1,2, \ldots, n, j=1,2, \ldots, v$, and we will obtain analogous estimations with respect to $p_{n, \rho}^{(j)}\left(x_{k, n}\right), k=1,2, \ldots, n, j=1,2, \ldots, v$ in Theorems 1.6 and 1.7.

There are many properties of $P_{n, \rho^{*}}(t)=P_{n}\left(W_{\rho^{*}} ; t\right)$ with respect to $W_{\rho^{*}}(t), W \in \tilde{\mathcal{F}}_{v}$, $v=2,3, \ldots$ of Definition 2.3 in [4-6, 19-21]. They were obtained by transformations from the results in $[1,2]$. In this paper, we consider $w=\exp (-R) \in \tilde{\mathcal{L}}_{v}$ and $p_{n, \rho}(x)=p_{n}\left(w_{\rho} ; x\right)$. In [5] we got the estimations of $P_{n, \rho^{*}}^{(j)}\left(t_{k n}\right), k=1,2, \ldots, n, j=1,2, \ldots, v-1$ with the weight $W_{\rho^{*}}(t) \in \tilde{\mathscr{F}}_{\nu}$. By a transformation of the results with respect to $P_{n, \rho^{*}}(t)$, we estimate $p_{n, \rho}^{(j)}\left(x_{k n}\right)$, $k=1,2, \ldots, n, j=1,2, \ldots, v-1$. In order to it we will give the transformation theorems in this section. In the following, we will give some applications of them.

Theorem 2.4 (see [21, Theorem 2.1]). Let $W(t)=w(x)$ with $x=t^{2}$. Then, the orthonormal polynomials $P_{n, \rho^{*}}(t)$ on $\mathbb{R}$ can be entirely reduced to the orthonormal polynomials $p_{n, \rho}(x)$ in $\mathbb{R}^{+}$as follows: for $n=0,1,2, \ldots$,

$$
P_{2 n, 2 \rho+(1 / 2)}(t)=p_{n, \rho}(x), \quad P_{2 n+1,2 \rho-(1 / 2)}(t)=t p_{n, \rho}(x) .
$$

In this paper, we will use the fact that $w_{\rho}(x)=x^{\rho} \exp (-R(x))$ is transformed into $W_{2 \rho+1 / 2}(t)=|t|^{2 \rho+1 / 2} \exp (-Q(t))$ as meaning that

$$
\begin{aligned}
\int_{0}^{\infty} p_{n, \rho}(x) p_{m, \rho}(x) w_{\rho}^{2}(x) d x & =2 \int_{0}^{\infty} p_{n, \rho}\left(t^{2}\right) p_{m, \rho}\left(t^{2}\right) t^{4 \rho+1} W^{2}(t) d t \\
& =\int_{-\infty}^{\infty} P_{2 n, 2 \rho+1 / 2}(t) P_{2 m, 2 \rho+1 / 2}(t) W_{2 \rho+1 / 2}^{2}(t) d t .
\end{aligned}
$$

Theorem 2.5. Let $Q(t)=R(x), x=t^{2}$. Then one has

$$
w(x)=\exp (-R(x)) \in \tilde{\mathcal{L}}_{v} \Longrightarrow W(t)=\exp (-Q(t)) \in \tilde{\mathscr{F}}_{v} .
$$


In particular, one has

$$
Q^{(v)}(t) \leqslant C\left(\frac{1}{t}\right)^{\delta}
$$

where $0 \leqslant \delta<1$ is defined in (1.13).

Proof. Let $w \in \tilde{\mathcal{L}}_{2}$. Then, from Lemma 2.2, one has $W \in \mathcal{F}\left(C^{2}+\right)$. Let $[x]$ denote the maximum integer as $[x] \leqslant x$ (Gaussian symbol). For $1 \leqslant j \leqslant v$, one has

$$
Q^{(j)}(t)=\sum_{i=0}^{[j / 2]} c_{i j} R^{(j-i)}(x) t^{j-2 i}, \quad c_{i j}>0(i=0,1, \ldots,[j / 2]), x=t^{2}
$$

Therefore, we easily see that (a) of Definition 2.3 holds. Let $x=t^{2}$. Since $R^{(\ell)}(x)$ is increasing for $x>0$ and $\ell=0,1, \ldots, v-1$, there exists $\xi$ with $0<\xi<x$ such that for $k=0,1, \ldots, v-2$,

$$
\frac{R^{(k)}(x)}{x}=R^{(k+1)}(\xi) \leqslant C R^{(k+1)}(x) .
$$

Then, since for $0 \leqslant k<j \leqslant v-1$,

$$
R^{(k)}(x) \leqslant C x^{j-k} R^{(j)}(x),
$$

one has by (b) of Definition 1.3 that

$$
\begin{aligned}
Q^{(j)}(t) & =\sum_{i=0}^{[j / 2} c_{i j} R^{(j-i)}(x) t^{j-2 i} \leqslant C R^{(j)}(x) t^{j} \leqslant C R^{(j-1)}(x) t^{j-1}\left(\frac{t R^{\prime}(x)}{R(x)}\right) \\
& \leqslant C Q^{(j-1)}(t) \frac{Q^{\prime}(t)}{Q(t)}, \quad 1 \leqslant j \leqslant v-1 .
\end{aligned}
$$

Similarly, one has by (2.16), (d) of Definition 1.2, and (b) of Definition 1.3 that

$$
\begin{aligned}
Q^{(v)}(t) & =c_{0, v} R^{(v)}(x) t^{\nu}+\sum_{i=1}^{[v / 2]} c_{i, v} R^{(v-i)}(x) t^{\nu-2 i} \\
& \leqslant c_{0, v} R^{(v)}(x) t^{\nu}+C R^{(v-1)}(x) t^{\nu-2} \\
& \leqslant C R^{(v-1)}(x) t^{\nu-1}\left(\frac{t R^{\prime}(x)}{R(x)}\right) \\
& \leqslant C Q^{(v-1)}(t) \frac{Q^{\prime}(t)}{Q(t)}
\end{aligned}
$$


Journal of Inequalities and Applications

Consequently, one has (b) in Definition 2.3. We know that

$$
\sum_{i=1}^{[v / 2]} c_{i, \nu} R^{(v-i)}(x) t^{\nu-2 i} \leqslant C, \quad t \in\left(0, c_{1}\right),
$$

and since $t^{\nu-\delta} \leqslant C$ on $t \in\left(0, c_{1}\right)$, one has from (1.13) that

$$
R^{(v)}(x) t^{v} \leqslant C\left(\frac{1}{t^{2}}\right)^{\delta} t^{v} \leqslant C\left(\frac{1}{t^{2}}\right)^{\delta} t^{\delta} \leqslant C\left(\frac{1}{t}\right)^{\delta} .
$$

Therefore, one has by (2.16)

$$
Q^{(v)}(t) \leqslant C\left(\frac{1}{t}\right)^{\delta}
$$

where $0 \leqslant \delta<1$ is defined in (1.13). The inequalities (d1) and (d2) of Definition 2.3 follow easily from (d1) and (d2) of Definition 1.3. Therefore, one has (2.14).

\section{Proofs of Theorems $1.5,1.6$, and 1.7}

For convenience, in the rest of this paper, we put as follows:

$$
\rho>-\frac{1}{2}, \quad \rho^{*}:=2 \rho+\frac{1}{2}, \quad p_{n}(x):=p_{n, \rho}(x), \quad P_{n}(t):=P_{n, \rho^{*}}(t),
$$

and $x_{k n}=x_{k, n, \rho}, t_{k n}=t_{k, n, \rho^{*}}$. Then, we know that $\rho^{*}>-1 / 2$ and

$$
p_{n}(x)=P_{2 n}(t), \quad x=t^{2}, \quad x_{k n}=t_{k, 2 n^{\prime}}^{2} \quad t_{k, 2 n}>0, k=1,2, \ldots, n .
$$

In the following, we introduce some useful notations.

(a) The Mhaskar-Rahmanov-Saff numbers $a_{v}$ and $a_{u}^{*}$ are defined as the positive roots of the following equations:

$$
\begin{array}{ll}
v=\frac{1}{\pi} \int_{0}^{1} a_{v} t R^{\prime}\left(a_{v} t\right)\{t(1-t)\}^{-1 / 2} d t, & v>0, \\
u=\frac{2}{\pi} \int_{0}^{1} a_{u}^{*} t Q^{\prime}\left(a_{u}^{*} t\right)\left(1-t^{2}\right)^{-1 / 2} d t, \quad u>0 .
\end{array}
$$

(b) Let

$$
\eta_{n}=\left\{n T\left(a_{n}\right)\right\}^{-2 / 3}, \quad \eta_{n}^{*}=\left\{n T^{*}\left(a_{n}^{*}\right)\right\}^{-2 / 3} .
$$

Then, one has the following. 
Lemma 3.1 (see $[1,(2.5),(2.7),(2.9)])$.

$$
a_{n}=a_{2 n}^{*}, \quad \eta_{n}=4^{2 / 3} \eta_{2 n}^{*}, \quad T\left(a_{n}\right)=\frac{1}{2} T^{*}\left(a_{2 n}^{*}\right) .
$$

To prove Theorem 1.6, we need some lemmas as follows.

Lemma 3.2 (see [21, Theorem 2.2, Lemma 3.7]). For the minimum positive zero $t_{[n / 2], n}([n / 2]$ is the largest integer $n / 2)$, one has

$$
t_{[n / 2], n} \sim a_{n}^{*} n^{-1},
$$

and for the maximum zero $x_{1 n}$, one has for large enough $n$,

$$
1-\frac{t_{1 n}}{a_{n}^{*}} \sim \eta_{n}^{*}, \quad \eta_{n}^{*}=\left(n T^{*}\left(a_{n}^{*}\right)\right)^{-2 / 3} .
$$

Moreover, for some constant $0<\varepsilon \leqslant 2$, one has

$$
T^{*}\left(a_{n}^{*}\right) \leqslant C n^{2-\varepsilon} .
$$

Lemma 3.3 (see [6, Theorem 2.5]). Let $W \in \mathcal{F}\left(C^{2}+\right)$ and $r=1,2, \ldots$. Then, uniformly for $1 \leqslant$ $k \leqslant n$,

$$
\left|\frac{P_{n, \rho}^{(r)}\left(t_{k, \rho, n}\right)}{P_{n, \rho}^{\prime}\left(t_{k, \rho, n}\right)}\right| \leqslant C\left(\frac{n}{\sqrt{a_{2 n}^{*}-t_{k, \rho, n}^{2}}}\right)^{r-1}
$$

Lemma 3.4 (see [5, Theorem 3.6 and Lemma 3.7 (3.20)]). Let $\rho^{*}>-1 / 2$ and $W(x)=$ $\exp (-Q(x)) \in \tilde{\boldsymbol{f}}_{v}, v \geqslant 2$. Assume that $1+2 \rho^{*}-\delta^{*} \geqslant 0$ for $\rho^{*}<0$ and if $T^{*}(t)$ is bounded, then assume

$$
a_{n}^{*} \leqslant C n^{1 /\left(1+v-\delta^{*}\right)}
$$

where $0 \leqslant \delta^{*}<1$ is defined in (2.8). If $t_{k n} \neq 0$, then one has for $j=1,2, \ldots, v$

$$
\left|P_{n}^{(j)}\left(t_{k n}\right)\right| \leqslant C\left(\frac{n}{a_{2 n}^{*}-\left|t_{k n}\right|}+\frac{T^{*}\left(a_{n}^{*}\right)}{a_{n}^{*}}\right)^{j-1}\left|P_{n}^{\prime}\left(t_{k n}\right)\right|,
$$

and in particular, if $j$ is even, then

$$
\left|P_{n}^{(j)}\left(t_{k n}\right)\right| \leqslant C\left(\frac{T^{*}\left(a_{n}^{*}\right)}{a_{n}^{*}}+\left|Q^{\prime}\left(t_{k n}\right)\right|+\frac{1}{\left|t_{k n}\right|}\right)\left(\frac{n}{a_{2 n}^{*}-\left|t_{k n}\right|}+\frac{T^{*}\left(a_{n}^{*}\right)}{a_{n}^{*}}\right)^{j-2}\left|P_{n}^{\prime}\left(t_{k n}\right)\right| .
$$


Remark 3.5. Let $W(t) \in \mathcal{F}\left(C^{2}+\right)$. Then, from [19, Theorem 1.6] we know that when $T^{*}(t)$ is unbounded, for any $\eta>0$, there exists $C(\eta)>0$ such that for $t \geq 1$,

$$
a_{t}^{*} \leqslant C(\eta) t^{\eta}
$$

In addition, since $T(x)=T^{*}(t) / 2$, we know that

(i) $T(x)$ is bounded $\Leftrightarrow T^{*}(t)$ is bounded,

(ii) $T(x)$ is unbounded $\Rightarrow a_{n} \leqslant C n^{\eta}$ for any $\eta>0$,

(iii) $T\left(a_{n}\right) \leqslant C n^{2-\varepsilon}$ for some constant $0<\varepsilon \leqslant 2$.

Lemma 3.6. For $j=1,2,3, \ldots$, one has

$$
p_{n}^{(j)}(x)=\sum_{i=1}^{j}(-1)^{j-i} c_{j, i} P_{2 n}^{(i)}(t) t^{-2 j+i}
$$

where $c_{j, i}>0$ satisfy that for $k=1,2, \ldots$,

$$
c_{k+1,1}=\frac{2 k-1}{2} c_{k, 1}, \quad c_{k+1, k+1}=\frac{1}{2^{k+1}}, \quad c_{1,1}=\frac{1}{2}
$$

and for $2 \leqslant i \leqslant k$

$$
c_{k+1, i}=\frac{c_{k, i-1}+(2 k-i) c_{k, i}}{2}
$$

Proof. It is easily proved, using the mathematical induction on $j$.

Proof of Theorem 1.5. By Lemmas 3.3, 3.6 and (3.2), one has

$$
\begin{aligned}
\left|p_{n}^{(j)}\left(x_{k n}\right)\right| & \leqslant C \sum_{i=1}^{j}\left|P_{2 n}^{(i)}\left(t_{k, 2 n}\right)\right|\left|t_{k, 2 n}^{-2 j+i}\right| \\
& \leqslant C \sum_{i=1}^{j}\left(\frac{2 n}{\sqrt{a_{4 n}^{*}{ }^{2}-t_{k, 2 n}^{2}}}\right)^{i-1}\left|P_{2 n}^{\prime}\left(t_{k, 2 n}\right)\right|\left|t_{k, 2 n}^{-2 j+i}\right| .
\end{aligned}
$$


Since by Lemma 3.2

$$
\begin{aligned}
\sum_{i=1}^{j}\left(\frac{2 n}{\sqrt{a_{4 n}^{* 2}-t_{k, 2 n}^{2}}}\right)^{i-1}\left|t_{k, 2 n}^{i-1}\right| & \leqslant C\left\{1+\left(\frac{2 n}{\sqrt{a_{4 n}^{*}{ }^{2}-t_{k, 2 n}^{2}}}\right)^{j-1}\left|t_{k, 2 n}^{j-1}\right|\right\} \\
& \leqslant C\left\{\left|t_{k, 2 n}^{-j+1}\right|+\left(\frac{2 n}{\sqrt{a_{4 n}^{*}{ }^{2}-t_{k, 2 n}^{2}}}\right)^{j-1}\right\}\left|t_{k, 2 n}^{j-1}\right| \\
& \leqslant C\left(\frac{n}{\sqrt{a_{4 n}^{*}{ }^{2}-t_{k, 2 n}^{2}}}\right)^{j-1}\left|t_{k, 2 n}^{j-1}\right|,
\end{aligned}
$$

one has from Lemma 3.1 that

$$
\begin{aligned}
\left|p_{n}^{(j)}\left(x_{k n}\right)\right| & \leqslant C\left(\frac{n}{\sqrt{a_{4 n}^{*}{ }^{2}-t_{k, 2 n}^{2}}}\right)^{j-1}\left|P_{2 n}^{\prime}\left(t_{k, 2 n}\right)\right|\left|t_{k, 2 n}^{-j}\right| \\
& \leqslant C\left(\frac{n}{\sqrt{a_{2 n}-x_{k, n}}}\right)^{j-1} x_{k, n}^{-(j-1) / 2}\left|p_{n}^{\prime}\left(x_{k, n}\right)\right| .
\end{aligned}
$$

Proof of Theorem 1.6. Since $w(x) \in \tilde{\mathcal{L}}_{v}$, we know that $W(t) \in \tilde{\mathcal{F}}_{v}$ and we know that $\delta^{*}=\delta$ by Theorem 2.5 and from (3.1), (3.2), and Lemma 3.1 that

(i) $\rho>-1 / 2 \Rightarrow \rho^{*}>-1 / 2$,

(ii) $1+2 \rho-\delta / 2 \geqslant 0$ for $\rho<-1 / 4 \Rightarrow 1+2 \rho^{*}-\delta^{*} \geqslant 0$ for $\rho^{*}<0$,

(iii) $a_{n} \leqslant C n^{2 /(1+v-\delta)} \Rightarrow a_{n}^{*} \leqslant C n^{1 /\left(1+v-\delta^{*}\right)}$.

Then, using Remark 3.5, we can apply Lemma 3.4 to $p_{n}(x)=P_{2 n, \rho^{*}}(t), x=t^{2}$. In a similar way to the proof of Theorem 1.5, one has from Lemma 3.4 and Lemma 3.1

$$
\begin{aligned}
\left|p_{n}^{(j)}\left(x_{k n}\right)\right| & \leqslant C \sum_{i=1}^{j}\left|P_{2 n}^{(i)}\left(t_{k, 2 n}\right)\right|\left|t_{k, 2 n}^{-2 j+i}\right| \\
& \leqslant C \sum_{i=1}^{j}\left(\frac{n}{a_{4 n}^{*}-\left|t_{k, 2 n}\right|}+\frac{T^{*}\left(a_{2 n}^{*}\right)}{a_{2 n}^{*}}\right)^{i-1}\left|P_{2 n}^{\prime}\left(t_{k, 2 n}\right)\right|\left|t_{k, 2 n}^{-2 j+i}\right| \\
& \leqslant C\left(\frac{n}{a_{4 n}^{*}-\left|t_{k, 2 n}\right|}+\frac{T^{*}\left(a_{2 n}^{*}\right)}{a_{2 n}^{*}}\right)^{j-1}\left|P_{2 n}^{\prime}\left(t_{k, 2 n}\right)\right|\left|t_{k, 2 n}^{-j}\right| \\
& \leqslant C\left(\frac{n}{\sqrt{a_{2 n}}-\sqrt{x_{k, n}}}+\frac{T\left(a_{n}\right)}{\sqrt{a_{n}}}\right)^{j-1} x_{k, n}^{-(j-1) / 2}\left|p_{n}^{\prime}\left(x_{k, n}\right)\right| .
\end{aligned}
$$


Journal of Inequalities and Applications

Let $j$ be even. Then, one has from Lemma 3.4 that

$$
\begin{aligned}
\sum_{i: \text { even }}\left|P_{2 n}^{(i)}\left(t_{k, 2 n}\right)\right|\left|t_{k, 2 n}^{-2 j+i}\right| \leqslant & C\left(\frac{T^{*}\left(a_{2 n}^{*}\right)}{a_{2 n}^{*}}+\left|Q^{\prime}\left(t_{k, 2 n}\right)\right|+\frac{1}{\left|t_{k, 2 n}\right|}\right)\left|P_{2 n}^{\prime}\left(t_{k, 2 n}\right)\right|\left|t_{k, 2 n}^{-2 j+2}\right| \\
& \times \sum_{i: \text { even }}\left(\frac{n}{a_{4 n}^{*}-\left|t_{k, 2 n}\right|}+\frac{T^{*}\left(a_{2 n}^{*}\right)}{a_{2 n}^{*}}\right)^{i-2}\left|t_{k, 2 n}^{i-2}\right|
\end{aligned}
$$

Since by Lemma 3.2 and

$$
\sum_{i: \text { even }}\left(\frac{n}{a_{4 n}^{*}-\left|t_{k, 2 n}\right|}+\frac{T^{*}\left(a_{2 n}^{*}\right)}{a_{2 n}^{*}}\right)^{i-2}\left|t_{k, 2 n}^{i-2}\right| \leqslant C\left(\frac{n}{a_{4 n}^{*}-\left|t_{k, 2 n}\right|}+\frac{T^{*}\left(a_{2 n}^{*}\right)}{a_{2 n}^{*}}\right)^{j-2}\left|t_{k, 2 n}^{j-2}\right|,
$$

one has

$$
\begin{aligned}
\sum_{i: \text { even }}\left|P_{2 n}^{(i)}\left(t_{k, 2 n}\right)\right|\left|t_{k, 2 n}^{-2 j+i}\right| \leqslant & C\left(\frac{T^{*}\left(a_{2 n}^{*}\right)}{a_{2 n}^{*}}+\left|Q^{\prime}\left(t_{k, 2 n}\right)\right|+\frac{1}{\left|t_{k, 2 n}\right|}\right)\left|P_{2 n}^{\prime}\left(t_{k, 2 n}\right)\right|\left|t_{k, 2 n}^{-j}\right| \\
& \times\left(\frac{n}{a_{4 n}^{*}-\left|t_{k, 2 n}\right|}+\frac{T^{*}\left(a_{2 n}^{*}\right)}{a_{2 n}^{*}}\right)^{j-2}, \\
\sum_{i: \text { odd }}^{j-1}\left|P_{2 n}^{(i)}\left(t_{k, 2 n}\right)\right|\left|t_{k, 2 n}^{-2 j+i}\right| \leqslant & C\left(\frac{n}{a_{4 n}^{*}-\left|t_{k, 2 n}\right|}+\frac{T^{*}\left(a_{2 n}^{*}\right)}{a_{2 n}^{*}}\right)^{j-2}\left|P_{2 n}^{\prime}\left(t_{k, 2 n}\right)\right|\left|t_{k, 2 n}^{-j-1}\right| \\
\leqslant & C\left(\frac{T^{*}\left(a_{2 n}^{*}\right)}{a_{2 n}^{*}}+\left|Q^{\prime}\left(t_{k, 2 n}\right)\right|+\frac{1}{\left|t_{k, 2 n}\right|}\right) \\
& \times\left(\frac{n}{a_{4 n}^{*}-\left|t_{k, 2 n}\right|}+\frac{T^{*}\left(a_{2 n}^{*}\right)}{a_{2 n}^{*}}\right)^{j-2}\left|P_{2 n}^{\prime}\left(t_{k, 2 n}\right)\right|\left|t_{k, 2 n}^{-j}\right| .
\end{aligned}
$$

Therefore, when $j$ is even, one has by Lemma 3.1 that

$$
\begin{aligned}
\left|p_{n}^{(j)}\left(x_{k n}\right)\right| \leqslant & C\left(\frac{T^{*}\left(a_{2 n}^{*}\right)}{a_{2 n}^{*}\left|t_{k, 2 n}\right|}+\frac{\left|Q^{\prime}\left(t_{k, 2 n}\right)\right|}{\left|t_{k, 2 n}\right|}+\frac{1}{t_{k, 2 n}^{2}}\right) \\
& \times\left(\frac{n}{a_{4 n}^{*}-\left|t_{k, 2 n}\right|}+\frac{T^{*}\left(a_{2 n}^{*}\right)}{a_{2 n}^{*}}\right)^{j-2}\left|P_{2 n}^{\prime}\left(t_{k, 2 n}\right)\right|\left|t_{k, 2 n}^{-j+1}\right| \\
\leqslant & C\left(\frac{T\left(a_{n}\right)}{\sqrt{a_{n} x_{k, n}}}+R^{\prime}\left(x_{k, n}\right)+\frac{1}{x_{k, n}}\right) \\
& \times\left(\frac{n}{\sqrt{a_{2 n}}-\sqrt{x_{k, n}}}+\frac{T\left(a_{n}\right)}{\sqrt{a_{n}}}\right)^{j-2} x_{k, n}^{-(j-2) / 2}\left|p_{n}^{\prime}\left(x_{k, n}\right)\right| .
\end{aligned}
$$

Next, we will prove Theorem 1.7. To prove it, we need two lemmas as follows. 
Lemma 3.7 ([5, Theorem 3.3]). Let $W(x)=\exp (-Q(x)) \in \tilde{\boldsymbol{F}}_{v}, v \geqslant 2$. Let $(1 / \varepsilon)\left(a_{n}^{*} / n\right) \leqslant\left|t_{k n}\right| \leqslant$ $\varepsilon a_{n}^{*}, 0<\varepsilon<1 / 2$, and $s=1,2, \ldots,(v-1) / 2$. Then, under the same conditions as the assumptions of Lemma 3.4, there exist $\beta(n, k), 0<D_{1} \leqslant \beta(n, k) \leqslant D_{2}$ for absolute constants $D_{1}, D_{2}$ such that the following equality holds:

$$
P_{n}^{(2 s+1)}\left(t_{k n}\right)=(-1)^{s} \beta_{n}^{s}(n, k)\left(\frac{n}{a_{n}^{*}}\right)^{2 s}\left(1+\tilde{\rho}_{2 s+1}\left(\varepsilon, t_{k n}, n\right)\right) P_{n}^{\prime}\left(t_{k n}\right),
$$

and $\left|\tilde{\rho}_{2 s+1}\left(\varepsilon, t_{k n}, n\right)\right| \rightarrow 0$ as $n \rightarrow \infty$ and $\varepsilon \rightarrow 0$.

From Lemma 3.3, we easily have the following.

Lemma 3.8. Let $W \in \mathcal{F}\left(C^{2}+\right)$ and $j=1,2, \ldots$. Then, uniformly for $\left|t_{k n}\right| \leqslant a_{n}^{*} / 2$,

$$
\left|P_{n}^{(j)}\left(t_{k n}\right)\right| \leqslant C\left(\frac{n}{a_{n}^{*}}\right)^{j-1}\left|P_{n}^{\prime}\left(t_{k n}\right)\right|
$$

Proof of Theorem 1.7. By Lemmas 3.4, 3.6 and Theorem 2.4, one has

$$
\begin{aligned}
p_{n}^{(2 s+1)}\left(x_{k n}\right) & =\sum_{i=1}^{2 s+1}(-1)^{2 s+1-i} c_{2 s+1, i} P_{2 n}^{(i)}\left(t_{k, 2 n}\right) t_{k, 2 n}^{-2(2 s+1)+i} \\
& =\sum_{p=0}^{s} c_{2 s+1,2 p+1} P_{2 n}^{(2 p+1)}\left(t_{k, 2 n}\right) t_{k, 2 n}^{-4 s+2 p-1}-\sum_{p=1}^{s} c_{2 s+1,2 p} P_{2 n}^{(2 p)}\left(t_{k, 2 n}\right) t_{k, 2 n}^{-4 s+2 p-2} \\
& =: \sum_{\text {odd }}-\sum_{\text {even }}
\end{aligned}
$$

Since we know that

$$
\frac{1}{\varepsilon} \frac{a_{n}}{n^{2}} \leqslant x_{k, n} \leqslant \varepsilon a_{n} \Longrightarrow \frac{2}{\sqrt{\varepsilon}} \frac{a_{2 n}^{*}}{2 n} \leqslant\left|t_{k, 2 n}\right| \leqslant \sqrt{\varepsilon} a_{2 n^{\prime}}^{*} \quad 0<\sqrt{\varepsilon}<\frac{1}{2}
$$

by the same reason as the proof of Theorem 1.6, we can apply Lemma 3.7 to $P_{2 n}^{(2 p+1)}\left(t_{k, 2 n}\right)$. 
Journal of Inequalities and Applications

Then, using Lemmas 3.7 and 3.6, one has

$$
\begin{aligned}
\sum_{\text {odd }}= & \sum_{p=0}^{s} c_{2 s+1,2 p+1}(-1)^{p} \beta_{2 n}^{p}(2 n, k)\left(\frac{2 n}{a_{2 n}^{*}}\right)^{2 p}\left(1+\tilde{\rho}_{2 p+1}\right) P_{2 n}^{\prime}\left(t_{k, 2 n}\right) t_{k, 2 n}^{-4 s+2 p-1} \\
= & (-1)^{s} \beta_{2 n}^{s}(2 n, k)\left(\frac{n}{\sqrt{a_{n}}}\right)^{2 s}\left(\frac{1}{2}+\frac{\tilde{\rho}_{2 s+1}}{2}\right) P_{2 n}^{\prime}\left(t_{k, 2 n}\right) t_{k, 2 n}^{-2 s-1} \\
& +\sum_{p=0}^{s-1} c_{2 s+1,2 p+1}(-1)^{p} \beta_{2 n}^{p}(2 n, k)\left(\frac{2 n}{a_{2 n}^{*}}\right)^{2 p}\left(1+\tilde{\rho}_{2 p+1}\right) P_{2 n}^{\prime}\left(t_{k, 2 n}\right) t_{k, 2 n}^{-4 s+2 p-1} \\
= & (-1)^{s} \beta_{2 n}^{s}(2 n, k)\left(\frac{n}{\sqrt{a_{n}}}\right)^{2 s}\left(\frac{1}{2}+\frac{\tilde{\rho}_{2 s+1}}{2}\right) P_{2 n}^{\prime}\left(t_{k, 2 n}\right) t_{k, 2 n}^{-2 s-1} \\
& +(-1)^{s} \beta_{2 n}^{s}(2 n, k)\left(\frac{n}{\sqrt{a_{n}}}\right)^{2 s} P_{2 n}^{\prime}\left(t_{k, 2 n}\right) t_{k, 2 n}^{-2 s-1} \\
& \times \sum_{p=0}^{s-1} c_{2 s+1,2 p+1}(-1)^{p-s} 2^{2 s} \beta_{2 n}^{p-s}(2 n, k)\left(\frac{2 n}{a_{2 n}^{*}}\right)^{2(p-s)}\left(1+\tilde{\rho}_{2 p+1}\right) t_{k, 2 n}^{2(p-s)} .
\end{aligned}
$$

Here, $\tilde{\rho}_{2 p+1}:=\tilde{\rho}_{2 p+1}\left(\varepsilon, t_{k, 2 n}, 2 n\right), p=0,1, \ldots, s$. Since from (3.28) we see that for $0 \leqslant p \leqslant s-1$,

$$
\left(\frac{a_{2 n}^{*}}{2 n} \frac{1}{t_{k, 2 n}}\right)^{2(s-p)} \leqslant\left(\frac{\sqrt{\varepsilon}}{2}\right)^{2(s-p)} \leqslant\left(\frac{1}{4}\right)^{s-p} \varepsilon
$$

one has that

$$
\left|\sum_{p=0}^{s-1} c_{2 s+1,2 p+1}(-1)^{p-s} 2^{2 s} \beta_{2 n}^{p-s}(2 n, k)\left(\frac{a_{2 n}^{*}}{2 n} \frac{1}{t_{k, 2 n}}\right)^{2(s-p)}\left(1+\tilde{\rho}_{2 p+1}\right)\right| \longrightarrow 0,
$$

as $n \rightarrow \infty$ and $\varepsilon \rightarrow 0$. If we let

$$
\xi_{n, 1}^{\prime}\left(s ; x_{k n}\right):=\tilde{\rho}_{2 s+1}+\sum_{p=0}^{s-1} c_{2 s+1,2 p+1}(-1)^{p-s} 2^{2 s+1} \beta_{2 n}^{p-s}(2 n, k)\left(\frac{2 n}{a_{2 n}^{*}}\right)^{2(p-s)}\left(1+\tilde{\rho}_{2 p+1}\right) t_{k, 2 n}^{2(p-s)},
$$

then one has

$$
\begin{aligned}
\sum_{\text {odd }} & =(-1)^{s} \beta_{2 n}^{s}(2 n, k)\left(\frac{n}{\sqrt{a_{n}}}\right)^{2 s}\left(\frac{1}{2}+\frac{\xi_{n, 1}^{\prime}\left(s ; x_{k, n}\right)}{2}\right) P_{2 n}^{\prime}\left(t_{k, 2 n}\right) t_{k, 2 n}^{-2 s-1} \\
& =(-1)^{s} \beta_{2 n}^{s}(2 n, k)\left(\frac{n}{\sqrt{a_{n}}}\right)^{2 s}\left(1+\xi_{n, 1}^{\prime}\left(s ; x_{k, n}\right)\right) p_{n}^{\prime}\left(x_{k, n}\right) x_{k, n^{\prime}}^{-s}
\end{aligned}
$$


and $\left|\xi_{n, 1}^{\prime}\left(s ; x_{k n}\right)\right| \rightarrow 0$ as $n \rightarrow \infty$ and $\varepsilon \rightarrow 0$. On the other hand, we obtain

$$
\begin{aligned}
\sum_{\text {even }}= & \sum_{p=1}^{s} c_{2 s+1,2 p} \frac{P_{2 n}^{(2 p)}\left(t_{k, 2 n}\right)}{P_{2 n}^{\prime}\left(t_{k, 2 n}\right)} P_{2 n}^{\prime}\left(t_{k, 2 n}\right) t_{k, 2 n}^{-4 s+2 p-2} \\
= & \sum_{p=1}^{s} 2 c_{2 s+1,2 p} \frac{P_{2 n}^{(2 p)}\left(t_{k, 2 n}\right)}{P_{2 n}^{\prime}\left(t_{k, 2 n}\right)} p_{n}^{\prime}\left(x_{k, n}\right) t_{k, 2 n}^{-4 s+2 p-1} \\
= & (-1)^{s} \beta_{2 n}^{s}(2 n, k)\left(\frac{n}{\sqrt{a_{n}}}\right)^{2 s} p_{n}^{\prime}\left(x_{k, n}\right) x_{k, n}^{-s} \\
& \times \sum_{p=1}^{s} 2 c_{2 s+1,2 p}(-1)^{s} \beta_{2 n}^{-s}(2 n, k)\left(\frac{n}{a_{2 n}^{*}}\right)^{-2 s} \frac{P_{2 n}^{(2 p)}\left(t_{k, 2 n}\right)}{P_{2 n}^{\prime}\left(t_{k, 2 n}\right)} t_{k, 2 n}^{-2 s+2 p-1} \\
:= & \xi_{n, 2}^{\prime}\left(s ; x_{k, n}\right)(-1)^{s} \beta_{2 n}^{s}(2 n, k)\left(\frac{n}{\sqrt{a_{n}}}\right)^{2 s} p_{n}^{\prime}\left(x_{k, n}\right) x_{k, n}^{-s} .
\end{aligned}
$$

Here, one has from Lemma 3.8 and (3.28) that

$$
\begin{aligned}
\left|\xi_{n, 2}^{\prime}\left(s ; x_{k, n}\right)\right| & =\left|\sum_{p=1}^{s} 2 c_{2 s+1,2 p}(-1)^{s} \beta_{2 n}^{-s}(2 n, k)\left(\frac{n}{a_{2 n}^{*}}\right)^{-2 s} \frac{P_{2 n}^{(2 p)}\left(t_{k, 2 n}\right)}{P_{2 n}^{\prime}\left(t_{k, 2 n}\right)} t_{k, 2 n}^{-2 s+2 p-1}\right| \\
& \leqslant C \sum_{p=1}^{s}\left(\frac{a_{2 n}^{*}}{n} \frac{1}{t_{k, 2 n}}\right)^{2 s-2 p+1} \leqslant C \sqrt{\varepsilon} .
\end{aligned}
$$

Finally, if we let $\rho_{s}\left(\varepsilon, x_{k n}, n\right):=\xi_{n, 1}^{\prime}\left(s ; x_{k, n}\right)-\xi_{n, 2}^{\prime}\left(s ; x_{k, n}\right)$, then the result is proved.

\section{Proofs of Theorems $1.8,1.9$, and 1.10}

Lemma 4.1. Let $W(t) \in \mathcal{F}\left(C^{2}\right)$, and let $0<p<\infty$ and $\rho^{*}>-1 / 2$. Then, one has for $n \geqslant 1$ that

$$
\begin{aligned}
\left\|P_{n, \rho^{*}}(t) W(t)\left(|t|+\frac{a_{n}^{*}}{n}\right)^{\rho^{*}}\right\|_{L_{p}(\mathbb{R})} & \sim\left\|P_{n, \rho^{*}}(t) W(t)\left(|t|+\frac{a_{n}^{*}}{n}\right)^{\rho^{*}}\right\|_{L_{p}\left(a_{n}^{*} / 2 \leqslant|t| \leqslant 2 a_{n}^{*}\right)} \\
& \sim a_{n}^{* 1 / p-1 / 2} \begin{cases}1, & p<4, \\
\left\{\log \left(1+n T^{*}\left(a_{n}^{*}\right)\right)\right\}^{1 / 4}, & p=4, \\
\left\{n T^{*}\left(a_{n}^{*}\right)\right\}^{2 / 3(1 / 4-1 / p)}, & p>4 .\end{cases}
\end{aligned}
$$


Proof. In [21, theorem 2.6] we showed that

$$
\left\|P_{n, \rho^{*}}(t) W(t)\left(|t|+\frac{a_{n}^{*}}{n}\right)^{\rho^{*}}\right\|_{L_{p}(\mathbb{R})} \sim a_{n}^{* 1 / p-1 / 2} \begin{cases}1, & p<4 \\ \left\{\log \left(1+n T^{*}\left(a_{n}^{*}\right)\right)\right\}^{1 / 4}, & p=4 \\ \left\{n T^{*}\left(a_{n}^{*}\right)\right\}^{2 / 3(1 / 4-1 / p)}, & p>4 .\end{cases}
$$

But, seeing our proof of [21, Theorem 2.6] carefully, we can easily prove the first equivalence.

Lemma 4.2 (see [21, Theorem 2.4]). Let $W(t) \in \mathcal{F}\left(C^{2}\right), 0<p \leqslant \infty$ and $L \geqslant 0$. Let $\beta \in \mathbb{R}$. Then, given $r>1$, there exists a positive constant $C_{2}$ such that one has for any polynomial $P \in p_{n}$ that

$$
\left\|\left(P W_{\beta}\right)(t)\right\|_{L_{p}\left(a_{r n}^{*} \leqslant|t|\right)} \leqslant \exp \left(-C_{2} n^{\alpha}\right)\left\|\left(P W_{\beta}\right)(t)\right\|_{L_{p}\left(L\left(a_{n}^{*} / n\right) \leqslant|t| \leqslant a_{n}^{*}\left(1-L \eta_{n}\right)\right)} .
$$

Proof of Theorem 1.8. From Theorem 2.4 and Lemmas 4.2 and 4.1, one has

$$
\begin{aligned}
& \left\|\Theta_{n}^{1 / 4}(x) p_{n, \rho}(x) w(x)\left(x+\frac{a_{n}}{n^{2}}\right)^{\rho}\right\|_{L_{p}\left(\mathbb{R}^{+}\right)} \\
& \leqslant C\left\|\left(\frac{t^{2}}{a_{2 n}^{* 2}}\right)^{1 / 4} P_{2 n, \rho^{*}}(t) W(t)\left(t^{2}+\frac{a_{2 n}^{* 2}}{n^{2}}\right)^{\rho}|t|^{1 / p}\right\|_{L_{p}(\mathbb{R})} \\
& \leqslant C\left(\frac{1}{a_{2 n}^{*}}\right)^{1 / 2}\left\|P_{2 n, \rho^{*}}(t) W(t)\left(|t|+\frac{a_{2 n}^{*}}{n}\right)^{\rho^{*}}|t|^{1 / p}\right\|_{L_{p}\left(|t| \leqslant 2 a_{2 n}^{*}\right)} \\
& \leqslant C a_{2 n}^{*}{ }^{1 / p-1 / 2}\left\|P_{2 n, \rho^{*}}(t) W(t)\left(|t|+\frac{a_{2 n}^{*}}{n}\right)^{\rho^{*}}\right\| \\
& \sim a_{2 n}^{*} \|_{L_{p}(\mathbb{R})} \\
& \begin{cases}1, p-1 & p<4 \\
\left.\log \left(1+n T^{*}\left(a_{2 n}^{*}\right)\right)\right\}^{1 / 4}, & p=4 \\
\left\{n T^{*}\left(a_{2 n}^{*}\right)\right\}^{2 / 3(1 / 4-1 / p)}, & p>4 .\end{cases}
\end{aligned}
$$


On the other hand, one has by Theorem 2.4 and Lemma 4.1 that

$$
\begin{aligned}
& \left\|\Theta_{n}^{1 / 4}(x) p_{n, \rho}(x) w(x)\left(x+\frac{a_{n}}{n^{2}}\right)^{\rho}\right\|_{L_{p}\left(\mathbb{R}^{+}\right)} \\
& \geq\left\|\Theta_{n}^{1 / 4}\left(t^{2}\right) P_{2 n, \rho^{*}}(t) W(t)\left(t^{2}+\frac{a_{2 n}^{* 2}}{n^{2}}\right)^{\rho}|t|^{1 / p}\right\|_{L_{p}\left(a_{2^{*}} / 2 \leqslant|t| \leqslant 2 a_{2 n}^{*}\right)} \\
& \sim a_{2 n}^{*}{ }^{1 / p-1 / 2}\left\|P_{2 n, \rho^{*}}(t) W(t)\left(|t|+\frac{a_{2 n}^{*}}{n}\right)^{\rho^{*}}\right\|_{L_{p}\left(a_{2^{*}} / 2 \leqslant|t| \leqslant 2 a_{2 n}^{*}\right)} \\
& \sim a_{2 n}^{*}{ }^{2 / p-1} \begin{cases}1, & p<4, \\
\left\{\log \left(1+n T^{*}\left(a_{2 n}^{*}\right)\right)\right\}^{1 / 4}, & p=4, \\
\left\{n T^{*}\left(a_{2 n}^{*}\right)\right\}^{2 / 3(1 / 4-1 / p)}, & p>4 .\end{cases}
\end{aligned}
$$

Consequently, using Lemma 3.1, one has the result.

Lemma 4.3. Let $\rho>-1 / 2$, and let $w(x) \in \mathcal{L}\left(C^{2}+\right)$. Then, uniformly for $n \geq 1$ and $1 \leqslant j \leqslant n$, one has the following:

(a)

$$
\sup _{x \in I}\left|p_{n, \rho}(x) w(x)\right|\left(x+\frac{a_{n}}{n^{2}}\right)^{\rho}\left|\left(x+a_{n} n^{-2}\right)\left(a_{n}-x\right)\right|^{1 / 4} \sim 1
$$

(b) for $j \leqslant n-1$ and $x \in\left[x_{j+1, n}, x_{j n}\right]$,

$$
\left|p_{n, \rho}(x)\right| w(x)\left(x+\frac{a_{n}}{n^{2}}\right)^{\rho} \sim \min \left\{\left|x-x_{j n}\right|,\left|x-x_{j+1, n}\right|\right\} \varphi_{n}\left(x_{j n}\right)^{-1}\left[x_{j n}\left(a_{n}-x_{j n}\right)\right]^{-1 / 4},
$$

(c) for $1 \leqslant j \leqslant n-1$,

$$
x_{j n}-x_{j+1, n} \sim \varphi_{n}\left(x_{j n}\right),
$$

where

$$
\varphi_{u}(x)= \begin{cases}\frac{\sqrt{x+a_{u} u^{-2}}\left(a_{2 u}-x\right)}{u \sqrt{a_{u}-x+a_{u} \eta_{u}}}, & 0 \leqslant x \leqslant a_{u} \\ \varphi_{u}\left(a_{u}\right), & a_{u}<x .\end{cases}
$$

Proof. (a) It is from [1, Theorem 1.2]. (b) It is from [2, Theorem 1.3]. (c) It is from [2, Theorem 1.4]. 
Proof of Theorem 1.9. By Theorem 1.8, one has for $0<p \leqslant 4$,

$$
\begin{aligned}
\left\|p_{n, \rho}(x) w(x)\left(x+\frac{a_{n}}{n^{2}}\right)^{\rho}\right\|_{L_{p}\left(\mathbb{R}^{+}\right)} & \geqslant C\left\|\Theta_{n}^{1 / 4}(x) p_{n, \rho}(x) w(x)\left(x+\frac{a_{n}}{n^{2}}\right)^{\rho}\right\|_{L_{p}\left(\mathbb{R}^{+}\right)} \\
& \sim a_{n}{ }^{1 / p-1 / 2} \begin{cases}1, & p<4, \\
\left\{\log \left(n T\left(a_{n}\right)\right)\right\}^{1 / 4}, & p=4 .\end{cases}
\end{aligned}
$$

For $p>4$, we know by (4.7) and (4.8) that

$$
\begin{aligned}
& \int_{x_{n n}}^{a_{n} / 3}\left|p_{n, \rho}(x) w(x)\left(x+\frac{a_{n}}{n^{2}}\right)^{\rho}\right|^{p} d x \\
& \quad \geqslant C \sum_{x_{n n} \leqslant x_{j n} \leqslant a_{n} / 3} \int_{x_{j+1, n}}^{x_{j n}}\left|x-x_{j+1, n}\right|^{p} d x \varphi_{n}^{-p}\left(x_{j n}\right)\left[x_{j n}\left(a_{n}-x_{j n}\right)\right]^{-p / 4} \\
& \quad \sim a_{n}^{-p / 4} \sum_{x_{n n} \leqslant x_{j n} \leqslant a_{n} / 3} \varphi_{n}\left(x_{j n}\right) x_{j n}^{-p / 4} \\
& \sim a_{n}^{-p / 4} \int_{a_{n} n^{-2}}^{a_{n} / 3} t^{-p / 4} d t \\
& \sim a_{n}^{-p / 2+1} n^{2(p / 4-1)} .
\end{aligned}
$$

Then, for $p>4$

$$
\left\|p_{n, \rho}(x) w(x)\left(x+\frac{a_{n}}{n^{2}}\right)^{\rho}\right\|_{L_{p}\left(\mathbb{R}^{+}\right)} \geqslant C a_{n}^{-1 / 2+1 / p} n^{2(1 / 4-1 / p)} .
$$

Therefore, one has

$$
\left\|p_{n, \rho}(x) w(x)\left(x+\frac{a_{n}}{n^{2}}\right)^{\rho}\right\|_{L_{p}\left(\mathbb{R}^{+}\right)} \geqslant C a_{n}{ }^{1 / p-1 / 2} \begin{cases}1, & p<4 \\ \left\{\log \left(n T\left(a_{n}\right)\right)\right\}^{1 / 4}, & p=4 \\ n^{2(1 / 4-1 / p)}, & p>4 .\end{cases}
$$


On the other hand, one has from Theorem 1.8 that

$$
\begin{aligned}
\left\|p_{n, \rho}(x) w(x)\left(x+\frac{a_{n}}{n^{2}}\right)^{\rho}\right\|_{L_{p}\left(x \geq a_{n} / 3\right)} & \sim\left\|\Theta_{n}^{1 / 4}(x) p_{n, \rho}(x) w(x)\left(x+\frac{a_{n}}{n^{2}}\right)^{\rho}\right\|_{L_{p}\left(x \geq a_{n} / 3\right)} \\
& \leqslant\left\|\Theta_{n}^{1 / 4}(x) p_{n, \rho}(x) w(x)\left(x+\frac{a_{n}}{n^{2}}\right)^{\rho}\right\|_{L_{p}\left(\mathbb{R}^{+}\right)} \\
& \sim a_{n}{ }^{1 / p-1 / 2} \begin{cases}1, & p<4, \\
\left\{\log \left(n T\left(a_{n}\right)\right)\right\}^{1 / 4}, & p=4, \\
\left\{n T\left(a_{n}\right)\right\}^{2 / 3(1 / 4-1 / p)}, & p>4,\end{cases}
\end{aligned}
$$

and by (4.6) that

$$
\begin{aligned}
\left\|p_{n, \rho}(x) w(x)\left(x+\frac{a_{n}}{n^{2}}\right)^{\rho}\right\|_{L_{p}\left(x \leqslant a_{n} / 3\right)} & \leqslant C a_{n}^{-1 / 4}\left(\int_{0}^{a_{n} / 3}\left(x+\frac{a_{n}}{n^{2}}\right)^{-p / 4} d x\right)^{1 / p} \\
& \sim a_{n}{ }^{1 / p-1 / 2} \begin{cases}1, & p<4, \\
\left\{\log a_{n}\right\}^{1 / 4}, & p=4, \\
n^{2(1 / 4-1 / p)}, & p>4 .\end{cases}
\end{aligned}
$$

Therefore, one has

$$
\left\|p_{n, \rho}(x) w(x)\left(x+\frac{a_{n}}{n^{2}}\right)^{\rho}\right\|_{L_{p}\left(\mathbb{R}^{+}\right)} \sim a_{n}{ }^{1 / p-1 / 2} \begin{cases}1, & p<4, \\ \left\{\log \left(n T\left(a_{n}\right)\right)\right\}^{1 / 4}, & p=4, \\ n^{2(1 / 4-1 / p)}, & p>4 .\end{cases}
$$

From Remark 3.5(iii), we see that $n T\left(a_{n}\right)<C n^{3}$. So, consequently, one has the result.

Let

$$
\Phi_{n}^{*}(t)=\max \left\{\eta_{n}^{*}, 1-\frac{|t|}{a_{n}^{*}}\right\}, \quad \eta_{n}^{*}=\left\{n T^{*}\left(a_{n}^{*}\right)\right\}^{-2 / 3}
$$

Then, we obtain by Lemma 3.1 that

$$
\Phi_{n}(x) \sim \Phi_{2 n}^{*}(t), \quad x=t^{2}
$$


Journal of Inequalities and Applications

Lemma 4.4 (see [21, Theorem 2.7]). Let $W_{\rho^{*}} \in \mathcal{F}\left(C^{2}\right)$ and $\rho^{*}>-1 / 2$. For $0 \leqslant s \leqslant r$ and $n \geqslant 1$, one has

$$
\begin{aligned}
&\left\|\Phi_{n}^{*}(t)^{(r / 4-1 / p)^{+}}\left|P_{n, \rho^{*}}(t) W(t)\left(|t|+\frac{a_{n}^{*}}{n}\right)^{\rho^{*}}\right|^{s}\right\|_{L_{p}(\mathbb{R})} \\
& \sim\left\|\Phi_{n}^{*}(t)^{(r / 4-1 / p)^{+}}\left|P_{n, \rho^{*}}(t) W(t)\left(|t|+\frac{a_{n}^{*}}{n}\right)^{\rho^{*}}\right|^{s}\right\|_{L_{p}\left(a_{n}^{*} / 2 \leqslant|t| \leqslant 2 a_{n}^{*}\right)} \\
& \sim \begin{cases}a_{n}^{* 1 / p-r / 2} \log n, & s=r, 4 \leqslant p r<\infty, \\
a_{n}^{* 1 / p-s / 2}, & \text { otherwise. }\end{cases}
\end{aligned}
$$

Proof of Theorem 1.10. By Theorem 2.4, we can transform $p_{n, \rho}(x)$ on $\mathbb{R}^{+}$to $P_{2 n, \rho^{*}}(t)$ on $\mathbb{R}$.

$$
\begin{aligned}
& \left\|\Theta_{n}^{r / 4}(x) \Phi_{n}^{(r / 4-1 / p)^{+}}(x)\left|p_{n} w(x)\left(x+\frac{a_{n}}{n^{2}}\right)^{\rho}\right|^{S}\right\| \|_{L_{p}\left(\mathbb{R}^{+}\right)} \\
& \quad=\left\|\Theta_{n}^{r / 4}\left(t^{2}\right) \Phi_{2 n}^{*(r / 4-1 / p)^{+}}(t)\left|P_{2 n} W(t)\left(t^{2}+\frac{a_{2 n}^{* 2}}{n^{2}}\right)^{\rho}\right|^{S}(2|t|)^{1 / p}\right\|_{L_{p}(\mathbb{R})} .
\end{aligned}
$$

Using Lemma 4.4 and noting (3.1), one has

$$
\begin{aligned}
& \left\|\Theta_{n}^{r / 4}\left(t^{2}\right) \Phi_{2 n}^{*(r / 4-1 / p)^{+}}(t)\left|P_{2 n} W(t)\left(t^{2}+\frac{a_{2 n}^{* 2}}{n^{2}}\right)^{\rho}\right|^{S}(2|t|)^{1 / p}\right\|_{L_{p}(\mathbb{R})} \\
& \geqslant\left\|\Theta_{n}^{r / 4}\left(t^{2}\right) \Phi_{2 n}^{*(r / 4-1 / p)^{+}}(t)\left|P_{2 n} W(t)\left(t^{2}+\frac{a_{2 n}^{* 2}}{n^{2}}\right)^{\rho}\right|^{s}(2|t|)^{1 / p}\right\|_{L_{p}\left(a_{2 n}^{*} / 2 \leqslant|t| \leqslant 2 a_{2 n}^{*}\right)} \\
& \geqslant C\left\|\Phi_{2 n}^{*(r / 4-1 / p)^{+}}(t)\left|P_{2 n} W(t)\left(|t|+\frac{a_{2 n}^{*}}{n^{2}}\right)^{\rho^{*}}\right|^{s}|t|^{1 / p-s / 2}\right\|_{L_{p}\left(a_{2 n}^{*} / 2 \leqslant|t| \leqslant 2 a_{2 n}^{*}\right)} \\
& \sim a_{2 n}^{* 1 / p-s / 2}\left\|\Phi_{2 n}^{*(r / 4-1 / p)^{+}}(t)\left|P_{2 n} W(t)\left(|t|+\frac{a_{2 n}^{*}}{n^{2}}\right)^{\rho^{*}}\right|^{s}\right\|_{L_{p}\left(a_{2 n}^{*} / 2 \leqslant|t| \leqslant 2 a_{2 n}^{*}\right)} \\
& \sim \begin{cases}a_{2 n}^{* 2 / p-r} \log n, & \text { if } s=r, 4 \leqslant p r<\infty, \\
a_{2 n}^{* 2 / p-s}, & \text { otherwise. }\end{cases}
\end{aligned}
$$

On the other hand, by Lemma 4.2, we see

$$
\left\|\Theta_{n}^{r / 4}(x) \Phi_{2 n}^{*(r / 4-1 / p)^{+}}(t)\left|P_{2 n}(t) W(t)\left(t^{2}+\frac{a_{2 n}^{* 2}}{n^{2}}\right)^{\rho}\right|^{s}(2 t)^{1 / p}\right\|_{L_{p}\left(a_{2 n}^{*} \leqslant|t|\right)} \leqslant C \eta_{2 n}^{(r / 4-1 / p)^{+}} e^{-c n},
$$


where $c>0$ is a constant. Therefore, using Lemma 4.4 and noting (3.1) and the definition of $\Theta_{n}$, one has

$$
\begin{aligned}
& \left\|\Theta_{n}^{r / 4}\left(t^{2}\right) \Phi_{2 n}^{*(r / 4-1 / p)^{+}}(t)\left|P_{2 n} W(t)\left(t^{2}+\frac{a_{2 n}^{* 2}}{n^{2}}\right)^{\rho}\right|^{s}(2|t|)^{1 / p}\right\|_{L_{p}(\mathbb{R})} \\
& \leqslant C a_{2 n}^{*-r / 2}\left\|\Phi_{2 n}^{*(r / 4-1 / p)^{+}}(t)\left|P_{2 n} W(t)\left(|t|+\frac{a_{2 n}^{*}}{n}\right)^{\rho^{*}}\right|^{s}|t|^{1 / p+r / 2-s / 2}\right\|_{L_{p}\left(|t| \leqslant 2 a_{2 n}^{*}\right)} \\
& \leqslant C a_{2 n}^{* 1 / p-s / 2}\left\|\Phi_{2 n}^{*(r / 4-1 / p)^{+}}(t)\left|P_{2 n} W(t)\left(|t|+\frac{a_{2 n}^{*}}{n}\right)^{\rho^{*}}\right|^{s}\right\|_{L_{p}\left(|t| \leqslant 2 a_{2 n}^{*}\right)} \\
& \leqslant C a_{2 n}^{* 1 / p-s / 2}\left\|\Phi_{2 n}^{*(r / 4-1 / p)^{+}}(t)\left|P_{2 n} W(t)\left(|t|+\frac{a_{2 n}^{*}}{n}\right)^{\rho^{*}}\right|^{S}\right\|_{L_{p}(\mathbb{R})} \\
& \sim\left\{\begin{array}{l}
a_{2 n}^{* 2 / p-r} \log n, \quad \text { if } s=r, 4 \leqslant p r<\infty, \\
a_{2 n}^{* 2 / p-s}, \quad \text { otherwise. }
\end{array}\right.
\end{aligned}
$$

Therefore, one has

$$
\begin{aligned}
\left\|\Theta_{n}^{r / 4}(x) \Phi_{n}^{(r / 4-1 / p)^{+}}(x)\left|p_{n} w(x)\left(x+\frac{a_{n}}{n^{2}}\right)^{\rho}\right|^{s}\right\|_{L_{p}\left(\mathbb{R}^{+}\right)} \\
\sim \begin{cases}a_{2 n}^{* 2 / p-r} \log n, & \text { if } s=r, 4 \leqslant p r<\infty, \\
a_{2 n}^{* 2 / p-s}, & \text { otherwise, }\end{cases} \\
\sim \begin{cases}a_{n}^{1 / p-r / 2} \log n, & \text { if } s=r, 4 \leqslant p r<\infty, \\
a_{n}^{1 / p-s / 2}, & \text { otherwise. }\end{cases}
\end{aligned}
$$

\section{Acknowledgments}

The authors thank the referees for many kind suggestions and comments. H. S. Jung was supported by SEOK CHUN Research Fund, Sungkyunkwan University, 2010.

\section{References}

[1] E. Levin and D. Lubinsky, "Orthogonal polynomials for exponential weights $x^{2 \rho} e^{-2 Q(x)}$ on $[0, d)$, , Journal of Approximation Theory, vol. 134, no. 2, pp. 199-256, 2005.

[2] E. Levin and D. Lubinsky, "Orthogonal polynomials for exponential weights $x^{2 \rho} e^{-2 Q(x)}$ on $[0, d)-\mathrm{II}$, , Journal of Approximation Theory, vol. 139, no. 1-2, pp. 107-143, 2006.

[3] T. Kasuga and R. Sakai, "Orthonormal polynomials for generalized Freud-type weights and higherorder Hermite-Fejér interpolation polynomials," Journal of Approximation Theory, vol. 127, no. 1, pp. $1-38,2004$. 
[4] H. Jung and R. Sakai, "Specific examples of exponential weights," Korean Mathematical Society. Communications, vol. 24, no. 2, pp. 303-319, 2009.

[5] H. S. Jung and R. Sakai, "Derivatives of orthonormal polynomials and coefficients of Hermite-Fejér interpolation polynomials with exponential-type weights," Journal of Inequalities and Applications, vol. 2010, Article ID 816363, 29 pages, 2010.

[6] H. S. Jung and R. Sakai, "The Markov-Bernstein inequality and Hermite-Fejér interpolation for exponential-type weights," Journal of Approximation Theory, vol. 162, no. 7, pp. 1381-1397, 2010.

[7] T. Kasuga and R. Sakai, "Uniform or mean convergence of Hermite-Fejér interpolation of higher order for Freud weights," Journal of Approximation Theory, vol. 101, no. 2, pp. 330-358, 1999.

[8] T. Kasuga and R. Sakai, "Orthonormal polynomials with generalized Freud-type weights," Journal of Approximation Theory, vol. 121, no. 1, pp. 13-53, 2003.

[9] T. Kasuga and R. Sakai, "Orthonormal polynomials for Laguerre-type weights," Far East Journal of Mathematical Sciences, vol. 15, no. 1, pp. 95-105, 2004.

[10] T. Kasuga and R. Sakai, "Conditions for uniform or mean convergence of higher order HermiteFejér interpolation polynomials with generalized Freud-type weights," Far East Journal of Mathematical Sciences, vol. 19, no. 2, pp. 145-199, 2005.

[11] Y. Kanjin and R. Sakai, "Pointwise convergence of Hermite-Fejér interpolation of higher order for Freud weights," The Tohoku Mathematical Journal, vol. 46, no. 2, pp. 181-206, 1994.

[12] Y. Kanjin and R. Sakai, "Convergence of the derivatives of Hermite-Fejer interpolation polynomials of higher order based at the zeros of Freud polynomials," Journal of Approximation Theory, vol. 80, no. 3, pp. 378-389, 1995.

[13] R. Sakai, "Hermite-Fejér interpolation," in Approximation Theory, vol. 58, pp. 591-601, North-Holland, Amsterdam, The Netherlands, 1991.

[14] R. Sakai, "The degree of approximation of differentiable by Hermite interpolation polynomials," in Progress in Approximation Theory, P. Nevai and A. Pinkus, Eds., pp. 731-759, Academic Press, Boston, Mass, USA, 1991.

[15] R. Sakai, "Certain unbounded Hermite-Fejér interpolatory polynomial operators," Acta Mathematica Hungarica, vol. 59, no. 1-2, pp. 111-114, 1992.

[16] R. Sakai and P. Vértesi, "Hermite-Fejér interpolations of higher order. III," Studia Scientiarum Mathematicarum Hungarica, vol. 28, no. 1-2, pp. 87-97, 1993.

[17] R. Sakai and P. Vértesi, "Hermite-Fejér interpolations of higher order. IV," Studia Scientiarum Mathematicarum Hungarica, vol. 28, no. 3-4, pp. 379-386, 1993.

[18] E. Levin and D. S. Lubinsky, Orthogonal Polynomials for Exponential Weights, Springer, New York, NY, USA, 2001.

[19] H. S. Jung and R. Sakai, "Derivatives of integrating functions for orthonormal polynomials with exponential-type weights," Journal of Inequalities and Applications, vol. 2009, Article ID 528454, 22 pages, 2009.

[20] H. S. Jung and R. Sakai, "Inequalities with exponential weights," Journal of Computational and Applied Mathematics, vol. 212, no. 2, pp. 359-373, 2008.

[21] H. S. Jung and R. Sakai, "Orthonormal polynomials with exponential-type weights," Journal of Approximation Theory, vol. 152, no. 2, pp. 215-238, 2008. 